\title{
ON ANNIHILATORS IN JORDAN ALGEBRAS
}

\author{
ANTONIO FERNÁNDEZ LÓPEZ \\ Dedicated to the memory of Pere Menal
}

\begin{abstract}
In this paper we prove that a nondegenerate Jordan algebra satisfying the descending chain condition on the principal inner ideals, also satisfy the ascending chain condition on the annikilators of the principal inner ideals. We also study annihilators in Jordan algebras without nilpotent clements and in $J B$-algebras.
\end{abstract}

\section{Introduction}

The notion of annihilator introduccd by Zel'manov plays a fundamental role in some of the most important theorems in Jordan theory [21], [22], [24]. However, annihilators had already bcen considered by Topping $[20]$ in the particular context of $J W$-algebras, and later by Bunce [5] and Battaglia [2] in $J B$-algebras.

In this paper we study annihilators in Jordan algebras, stressing the relationship between the definition due to Zel'manov and the other rclated notions given by Bunce and Battaglia. We compute annihilators in special Jordan algebras in terms of classical annihilators in their associative cnvelopes and *-envelopes. Then we consider annihilators in prime non-degenerate Jordan algebras with nonzero socle, getting as a consequence that a nondegenerate Jordan algebra satisfying the descending chain condition on the principal inner ideals (equivalently, coinciding with its socle), also satisfies the maximality condition for the annihilators of the principal inner ideals.

The paper is organized as follows: In Section 1 we give the basic definitions and collect the identities that will be used throughout this paper. General properties of annihilators are settled in Section 2. Annihilators in special Jordan algebras are studied in Section 3. Section 4 deals with annihilators in prime nondegenerate Jordan algebras with nonzero socle. Section 5 concerns with annihilators in Jordan algebras without nilpotent elements and in $J B$-algebras. 


\section{Basic identities and definitions}

All the algebras we consider here are over a field $K$ of characteristic different from 2. A (nonassociative) algebra $J$ with product $x . y$ satisfying:

$$
\begin{aligned}
x \cdot y & =y \cdot x \\
x^{2} \cdot(y \cdot x) & =\left(x^{2} \cdot y\right) \cdot x \text { (Jordan identity) }
\end{aligned}
$$

is called a (linear) Jordan algebra (our standard references for Jordan algebras are [12], [25]. Every associative algebra $A$ gives rise to a Jordan algebra $A^{+}$under the new multiplication given by

$$
x . y=1 / 2(x y+y x) .
$$

The following expression relating the associator of three elements in the Jordan product to a double commutator will be frecuently used in what follows

$$
(x, y, z)^{+}=1 / 4[y,[x, z]]
$$

$(a, b, c)^{+}=(a . b) . c-a .(b . c)$ being the associator of $a, b, c$ in $A^{+}$, and $[a, b]=a b-b a$ the commutator of $a, b$ in $A$.

Jordan algebras which are subalgebras of a Jordan algebra $A^{+}$are called special Jordan algebras. For every associative algebra $A$ with involution $*: A \rightarrow A$ the set of all hermitian elements $H(A, *)=\{a \in A$ : $\left.a=a^{*}\right\}$ is a subalgebra of $A^{+}$, and therefore special. Another important class of special Jordan algebras is obtained as follows. Let $V$ be a vector space over a field $K$ with a symmetric bilincar form $\varphi: V \times V \rightarrow K$. Consider the vector space direct sum $J=K \ominus V$ and define

$$
(\alpha, x) \cdot(\beta, y)=(\alpha \beta+\varphi(x, y), \alpha y+\beta x) .
$$

Then $J$ is a special Jordan algebra. Actually $J$ is a Jordan subalgebra of the Clifford algebra $C(V, \varphi)$. If $\varphi$ is nondegenerate and $\operatorname{dim}_{K} V>1$, then $J$ is a simple Jordan algebra.

Every Jordan algebra which is not special is called an exceptional Jordon algebra. Let $C$ be a Cayley-Dickson algebra over $K(C$ is an 8-dimensional alternative algebra obtained by doubling a quaternion algebra by the Cayley-Dickson process). Then the set $H_{3}(C, \gamma)=$ $H\left(M_{3}(C), *\right)$ of all $3 \times 3$ matrices in $C$ which are hermitian under the involution $X^{*}=\gamma^{-1} \bar{X}^{t} \gamma\left(\gamma=\operatorname{diag}\left\{\gamma_{1}, \gamma_{2}, \gamma_{3}\right\}\right.$ for $\gamma_{i} \neq 0$ in $\left.K\right)$ is a simple 27-dimensional exceptional Jordan algebra.

In order to get identities in Jordan algebras is very useful the following theorem due to Macdonald that is usually used in the following form 
(1.6). Any polynomial identity in three variables with degree at most 1 in one variable, and which holds in all special Jordan algebras, holds in all Jordan algebras.

Consider the following triple product in any associative algebra $A$

$$
\{a b c\}=1 / 2(a b c+c b a) \text {. }
$$

It is not difficult to see that this can be expressed in terms of the Jordan product as follows

$$
\{a b c\}=(a . b) \cdot c+(c . b) \cdot a-(a . c) \cdot b .
$$

This is the definition of $\{a b c\}$ in a general Jordan algebra. We shall also write

$$
U_{a, c} b=\{a b c\}=L(a, b) c, \quad U_{a, a}=U_{a}=U(a) .
$$

Note that

$$
U_{a}=2 L_{a}^{2}-L_{a^{2}}
$$

where $L_{a} x=a . x$. The following identities can be casily verified in any special Jordan algebra, so by Macdonald's theorem they hold in a general Jordan algebra.

$$
\begin{aligned}
& U\left(U_{a} b\right)=U_{a} U_{b} U_{a} \\
& \left(U_{a} b\right)^{2}=U_{a} U_{b} a^{2} \\
& 4(a . t)^{2}=U_{a} t^{2}+U_{t} a^{2}+2 a . U_{t} a \\
& L(a, b)+L(b, a)=2 L_{a} \cdot b \\
& 2\left(L_{a}, L_{b}\right]=L(a, b)-L(b, a) \\
& L\left(U_{a} b, b\right)=L\left(a, U_{b} a\right) \\
& U_{a} t^{2}=2\{a . t t a\}-a . U_{t} a \\
& \left\{a U_{t}(a . x) x\right\}=2\{a\{a . t x t\} x\}-\left\{a a . U_{t} x x\right\} .
\end{aligned}
$$

Another way to get identities is by linearization. By linearizing Jordan identity (1.2) and (1.16) we get respectively

$$
\begin{aligned}
& (a . c, x, b)=(c, x, a . b)+(a, x, c . b) \\
& 2 L(\{a b c\}, b)=L\left(a, U_{b} c\right)+L\left(c, U_{b} a\right)
\end{aligned}
$$

where $(z, y, t)=(z . y) \cdot t-z \cdot(y \cdot t)=\left[L_{t}, L_{z}\right] y$ is the associator of $z, y, t$. 
The notion of invertibility in a unital associative algebra can be expressed in terms of the Jordan product. Indeed, let $A$ be an associative algebra with identity element 1 . An element $x \in A$ is invertible with inverse $y$ if and only if

$$
x \cdot y=1 \text { and } x^{2} \cdot y=x .
$$

Then this is the definition of invertible element in any unital Jordan algebra $J$. Each invertible element $x$ has a unique inverse $y$, and a unita. Jordan algebra $J$ in which each nonzero element is invertible is called a division Jordan algebra. It is clear from above that a unital associative algebra $A$ is a division algebra if and only if $A^{+}$is a Jordan division algebra. If $A$ has an involution $*$ then $H(A, *)$ is also a division Jordan algebra.

(1.22). Let $J=K \oplus V$ be the Jordan algebra of a symmetric bilinear form $\varphi$. Then an element $a=\varphi+x$ is invertible if and only if $\alpha^{2}-$ $\varphi(x, x) \neq 0$. In such case the inverse of $a$ is $b=\left(\alpha^{2}-\varphi(x, x)\right)^{-1}(\alpha-x)$.

For an invertible element $x$ in a unital Jordan algebra the multiplication operator $L_{x}$ is not necessarily invertible. Consider the division algebra of real quaternions and take the quaternions $i, j$. Then $L_{i} j=0$ since $i j=-j i$. However, in every unital Jordan algebra $J$, an element $x$ is invertible if and only if $U_{x}$ is invertible. Hence an invertible element $x$ is not a zero divisor: $U_{x} y=0 \Rightarrow y=0$.

\section{Annihilators. General theory}

Let $J$ be a Jordan algebra (over $K$ ) and let $J^{1}$ be its unital hull: $J^{1}=J$ if $J$ has identity element and $J^{1}=K \oplus J$ the unitization of $J$ otherwise.

Lemma. For $a, b$ in $J$ the following conditions are equivalent:

$$
\begin{aligned}
& \left\{a b J^{1}\right\}=0 \\
& \left\{b a J^{1}\right\}=0 \\
& a . b=0=(a, J, b) .
\end{aligned}
$$

Proof: By (1.8), $\{a b 1\}=a \cdot b=\{b a 1\}$, and by (1.14), $\{a b x\}+$ $\{b a x\}=2(a . b) . x\left(x \in J^{1}\right)$. Hence $(2.1) \Leftrightarrow(2.2)$. 
Since $2(a, x, b)=2\left[L_{b}, L_{a} \mid x=\{b a x\}-\{a b x\}\right.$ by (1.15), we have that $(2.1) \Rightarrow(2.3)$. Finally, if (2.3) holds we have by (1.14) and (1.15) that

$$
\begin{aligned}
& \{a b x\}+\{b a x\}=2 L_{a, b} x=0 \text { and } \\
& \{b a x\}-\{a b x\}=2\left[L_{b}, L_{a}\right] x=2(a, x, b)=0 .
\end{aligned}
$$

Hence $\{a b x\}=0=\{b a x\}$, which completes the proof.

Given $a \in J$ the set of all $b \in J$ satisfying the above equivalent conditions is called the annihilator of $a$ and it is denoted by Ann $(a)$. It follows from definition that $x^{2}=0$ implies $x \in \operatorname{Ann}(x)$, and that if $b \in \operatorname{Ann}(a)$ then $U_{a} b=0$. Hence every invertible element has zero annihilator. For any subset $M$ of $J$, the annihilator of $M$ is defined as the intersection of all $\operatorname{Ann}(a)(a \in M)$. We recall that a subspace $I$ of $J$ is called an ideal, inner ideal, strict inner ideal if $I, J \subset I, U_{I} J \subset I, U_{I} J^{1} \subset I$, respectively. Note that for any element $a$ in $J, U_{a} J$ is an inner ideal called the principal inner generated by $a$.

Proposition (Zel'manov). Let $J$ be a Jordan algebra, $a, b \in J$ and $M \subset J$. Then

$$
\begin{aligned}
& \operatorname{Ann}(M) \text { is a strict inner ideal of } J \\
& a \in \operatorname{Ann}(b) \Longleftrightarrow b \in \operatorname{Ann}(a) \\
& \operatorname{Ann}(\operatorname{Ann}(\operatorname{Ann} M))=\operatorname{Ann}(M) \\
& \text { If } M \text { is an ideal of } J \text { then } \operatorname{Ann}(M) \text { is also an ideal } \\
& \operatorname{Ann}(b) \subset \operatorname{Ann}\left(U_{b} a\right) \\
& \text { If } a \cdot b=a \cdot b^{2}=0 \text { then } b^{2} \in \operatorname{Ann}(a) .
\end{aligned}
$$

Proof: (2.4) Since the intersection of strict inner ideals is again a strict inner ideal, we need only to prove that $\operatorname{Ann}(a)$ is a strict inner ideal. Clearly Ann( $a$ ) is a subspace. Let $b \in \operatorname{Ann}(a)$ and $c \in J^{l}$. By (1.20)

$$
L\left(a, U_{b} c\right)=2 L(\{a b c\}, b)-L\left(c, U_{b} a\right)=0
$$

since $\{a b c\}=0$ and $U_{b} a=\{b a b\}=0$, so $U_{b} c \in \operatorname{Ann}(a)$.

(2.5) It follows from the symmetry of the definition: $(2.1) \Leftrightarrow(2.2)$.

(2.6) Since $M \subset \operatorname{Ann}(\operatorname{Ann} M)$ we have that $\operatorname{Ann}(\operatorname{Ann}(\operatorname{Ann} M)) \subset$ Ann $(M)$. The reverse inclusion is obvious.

(2.7) Suppose now that $M$ is an ideal of $J$, and let $a \in J, x \in \operatorname{Ann}(M)$. Then $a . x \in \operatorname{Ann}(M)$ since by $(2.3)$

$$
(a . x) \cdot m=x \cdot(a . m) \subset x . M=0
$$


and by (1.19)

$$
(a . x, b, m)=(x, b, a . m)+(a, b, x . m)=0
$$

for all $b \in J, m \in M$.

(2.8) $x \in \operatorname{Ann}(b) \Leftrightarrow$ (by (2.5) $b \in \operatorname{Ann}(x) \Rightarrow$ (by 2.4) $U_{b} a \in \operatorname{Ann}(x) \Leftrightarrow$ $x \in \operatorname{Ann}\left(U_{b} a\right)$.

(2.9) $a . b=a . b^{2}=0 \Rightarrow$ (by 1.19) $\left(b^{2}, x, a\right)=2(b, x, b . a)=0$, so $b^{2} \in \operatorname{Ann}(a)$ by (2.3).

An element $b \in J$ is called von Neumann regular if $b=U_{b} a$ for some $a \in J$. Since $U_{b} a=b a b$ in any special algebra $J$, we have that this definition agrees with the associative one. Since $\operatorname{Ann}(b) \subset \operatorname{Ann}\left(U_{b} a\right)$ by (2.8), we have

$$
\operatorname{Ann}(b)=\operatorname{Ann}\left(U_{b} J\right)
$$

for every von Neumann regular element $b$ in $J$. In particular if $e$ is an idempotent then

$$
\operatorname{Ann}(e)=\operatorname{Ann}\left(U_{e} J\right)=\{x \in J: x . e=0\}
$$

by Peirce relations [12, Lemma 1, p. 119].

The reader is referred to Zcl'manov's paper [24] for other results on annihilators. In fact, he gives the following suggestive characterization for the annihilator of a nondegenerate ideal $M$ of a Jordan algebra $J$ (a Jordan algebra is called nondegenerate if $U_{x}=0$ implies $x=0$; clearly an associative algebra $A$ is semiprime if and only if the Jordan algebra $A^{+}$is nondegenerate).

$$
\operatorname{Ann}(M)=\left\{a \in J: U_{a} M=0\right\} .
$$

for any nondegenerate ideal $M$ of $J$.

\section{Annihilators in special Jordan algebras}

Let $A$ be an associative algcbra and $J$ a special Jordan algebra $J \subset A^{+}$. For any subset $S$ of $J$ write $\operatorname{Ann}_{A}(S)$ to denote the usual annihilator of $S$ in $A$, i.e.,

$$
\operatorname{Ann}_{A}(S)=\{x \in A: x S=S x=0\}=\operatorname{Lan}(S) \cap \operatorname{Ran}(S)
$$

and $\operatorname{Ann}_{J}(S)$ to denote the annihilator of $S$ in $J$. By (1.4) and (2.3)

$$
\operatorname{Ann}_{J}(S)=\{a \in J: a . S=0=[J,[a, S]]\}
$$


Clearly $A_{A n n_{A}}(S) \cap J \subset A n_{J}(S)$ but in general this inclusion can be strict, even if $J=A^{+}$. Indeed, in the full matrix algebra $M_{3 \times 3}(\mathrm{H})$ of all $3 \times 3$ matrices over the real quaternions $\mathrm{H}$, consider the real subalgebra $A$ generated by the matrices $a=i\left(E_{21}+E_{32}\right), b=j\left(E_{21}+E_{32}\right)$ where $1, i, j, k$ is a canonical basis of $\mathrm{H}$ and $E_{t s}$ are the matrix units. Then $A$ is the four-dimensional algebra.

$$
A=\mathrm{R} a \oplus \mathrm{R} a^{2} \oplus \mathrm{R} b \oplus \mathrm{R} c
$$

satisfying $a b=c, a^{2}=b^{2}, b a=-c, a^{n} b^{m}=b^{n} a^{m}=0$ for all nonnegative integers $n, m$ with $n+m \geq 3$. Then it is not difficult to verify that $\operatorname{Ann}_{A}(a)=\mathrm{Ra}^{2} \oplus \mathrm{R} c$ but $\operatorname{Ann}_{J}(a)=\operatorname{Ann}_{A}(a) \oplus \mathrm{R} b$. Note that the above algebra $A$ is not semiprime; in fact $x A x=0$ for all $x \in A$. However, as it will be seen below, for a semiprime associative algebra both annihilators agree.

An associative algebra $A$ is an (associative) envelope for a special Jordan algebra $J \subset A^{+}$if it is generated as an associative algebra by the elements of $J$, and an associative algebra with involution $(A, *)$ is a *-envelope for $J$ if it is generated by $J \subset H(A, *)$. Notice that *envelopes for $J$ are in particular envelopes for $J$.

(3.2) Proposition. Let $J$ be a special Jordan algebra and $S$ a subset of $J$. If a semiprime associative algebra $A$ is an envelope for $J$ then $\operatorname{Ann}_{J}(S)=\operatorname{Ann}_{A}(S) \cap J$.

Proof: Since the inclusion $\operatorname{Ann}_{A}(S) \cap J \subset \operatorname{Ann}_{J}(S)$ holds for a general associative algebra $A$, we need only to prove the reverse inclusion. Let $a \in \operatorname{Ann}_{J}(S)$. By (3.1), for every $x \in S$ we have that $a . x=0$ and $[a, x] y=y[a, x](y \in J)$. Since $A$ is generated as an associative algebra by $J$, this implies that $[a, x] \in Z(A)$ (the centre of $A$ ). Hence $2 a x=$ $[a, x] \in Z(A)$. Then $a x a=a^{2} x=-a x a \Rightarrow(a x)^{2}=0 \Rightarrow a x=0$ since $Z(A)$ has no nonzero nilpotent elements by serniprimeness of $A$.

A Jordan algebra $J$ is said to be prime if $U_{T} S=0$ implies $T=0$ or $S=0, T, S$ ideals of $J$. By a result of Bresar [4], an associative algebra $A$ is prime iff

$$
\{a A b\}=0 \Rightarrow a=0 \text { or } b=0 .
$$

Hence $A$ is prime if and only if $A^{+}$is prime.

Recently, this characterization of primeness without involving ideals has been proved in $[3]$ for nondegenerate Jordan algebras.

The classification of all prime nondegenerate (linear) Jordan algebras was achieved by Zel'manov in [23]. Later McCrimmon and Zel'manov [16] extended the result to Jordan algebras over an arbitrary ring of scalars. 
(3.4) Zelmanov's Prime Theorem. The prime nondegenerate Jordan algebras are precisely

(i) Albert algebras: They are a central order in a simple 27-dimensional exceptional Jordon algebra,

(ii) special Jordan algebras of quadratic type: They are a central order in the simple Jordon algebra of a nondegenerate symmetric bilinear form,

(iii) hermitian algebras: They contain a hermitian ideal $\mathcal{H}=H(A, *)$ such that

$$
H(A, *)=\mathcal{H C} J \subset H(Q(A, *), *)
$$

where $(A, *)$ is a*-prime associative algebra with involution which is a *-envelope for the hermitian ideal $\mathcal{H}$, and $Q(A, *)$ is the symmetric Martindale *-algebra of quotients of $(A, *)$.

Moreover, either $A$ is prime in (izi) or

$$
B^{+}=r C J \subset Q(B)^{+}
$$

where $B$ is a prime associative algebra and $Q(B)$ is the symmetric Martindale algebra of quotients of $B$.

The reader is referred to [18], $[\mathbf{7}]$ : [15] for definition and properties of the symmetric Martindale algebra (*-algebra) of quoticnts. Nevertheless, we state here the following result that will be used below.

(3.5). Let $A$ be a prime associotive algebra and let $Q(A)$ be its symmetric Martindale algebro of quotients.

(i) For each $q \in Q(A)$ there is a nonzero ideal $M$ of $A$ such that $q M+M q \subset A$,

(ii) $q N=0$ (or $N q=0$ ) for some nonzero ideal $N$ of $A$ implies $q=0$,

(iii) $Q(A)$ is a prime ossociative algebra containing $A$.

(iv) Every involution on $A$ has a unique extension to $Q(A)$.

Now we compute annihilators in prime nondegenerate Jordan algebras of hermitian type.

(3.6) Proposition. Let J a prime nondegenerate Jordan algebra such that either

$$
A^{+}=M C J \subset Q(A)^{+}
$$

where $A$ is a prime associative olgebra, or

$$
H(A, *)=M \subset J \subset H(Q(A), *)
$$


where $(A, *)$ is a prime associative algebra with involution, which is a *-envelope for the ideal $M$. Then for any subset $S$ of $J$ we have

$$
\operatorname{Ann}_{J}(S)=\operatorname{Ann}_{Q(A)}(S) \cap J
$$

Proof: Again we must only prove the inclusion $A n_{J}(S) \subset \operatorname{Ann}_{Q(A)}(S)$. Let $a \in \operatorname{Ann}_{J}(S)$. Since $M$ generates $A$ as an associative algebra in both cases, we have as in the proof of (3.2) that for all $x \in S$, ax commutes with each element $b \in A$. Now let $q \in Q(A)$. By (3.5i), there exists a nonzero ideal $I$ of $A$ such that $q I+I q \subset A$. Hence for $t=a x$ and $y \in I$ we have

$$
(t q) y=(q y) t=(q t) y \Rightarrow[a, t] I=0
$$

and hence $[q, t]=0$ by (3.5ii). Then $t=a x$ belongs to the centre of $Q(A)$. Since $Q(A)$ is a prime associative algebra (3.5iii), we may conclude as in the proof of (3.2) that $a x=0$, as required.

\section{Annihilators in Jordan algebras with nonzero socle}

We recall that the socle $\operatorname{Soc}(J)$ of a nondegenerate Jordan algebra $J$ is defined to be the sum of all its minimal inner ideals. If $J$ contains minimal inner ideals then $\operatorname{Soc}(J)$ is a direct sum of simple ideals each of which contains a division idempotent $e\left(U_{e} J\right.$ is a division Jordan algebra) [17]. As it was shown in [6, Prop. 2.6], for a semiprime associative algebra $A$ the socle of the Jordan algebra $A^{+}$coincides with the (usual) socle of $A$, and if $A$ has an involution * then $\operatorname{Soc}(H(A, *))=H(\operatorname{Soc}(A), *)$.

(4.1) Proposition. Let $J$ be a Jordan algebra and $M$ and ideal of $J$ such that $M^{2}=M$ (this holds for instance if $M$ is von Neumann regular or if $M$ is a simple Jordan algebra). Then

$$
\operatorname{Ann}(M)=\{a \in J: a \cdot M=0\}
$$

If $J$ is nondegenerate then

(ii) $\operatorname{Ann}(\operatorname{Soc}(J))=\{a \in J:$ a.e $=0$ for all division idempotents $e \in J\}$

Proof: (i) Let $a \in J$ such that $a . M=0$. By (2.3) we must show that $(a, J, M)=0$; but, by $(1.19)$,

$$
(a, J, M)=(a, J, M \cdot M)=(a . M, J, M)-(M, J, a . M)=0 .
$$

(ii) Since $\operatorname{Soc}(J)$ is von Neumann regular (see [9]) $\operatorname{Soc}(J)^{2}=\operatorname{Soc}(J)$, and hence, by (i), $\operatorname{Ann}(\operatorname{Soc}(J))=\{a \in J: a: \operatorname{Soc}(J)=0\}$, but by Litoff 
theorem for Jordan algebras [1], for every $x \in \operatorname{Soc}(J), x \in U_{u} J$ where $u=e_{1}+\cdots+e_{n}$ is a sum of orthogonal division idempotents. Hence, by (2.11), $a \in \operatorname{Ann}(\operatorname{Soc}(J))$ if and only if $a . e=0$ for all division idempotents $e$ in $J$, which completes the proof.

Among other many interesting applications of Zel'manov's theorem for prime nondegenerate Jordan algebras, one may get, as it is shown in [7], the following theorem due to Osborn and Racine [17], but before we recall some definitions and notations that will be used later.

Following $[11$, p. 69], let $(X, Y,(.)$,$) be a pair of dual vector spaces$ over a division associative algebra $\Delta$, where $X$ is a left vector space, $Y$ a right vector space, and $(x, y)$ a nondegenerate bilinear mapping over $\Delta$. A linear operator $a: X \rightarrow X$ is said to be continuous if there exists $a^{\#}: Y \rightarrow Y$, necessarily unique, such that $(x a, y)=\left(x, a^{\#} y\right)$. Notice that we write mappings of a left vector space on the right (thus composing then from left to right) and mappings of a right vector space on the left (thus composing them from right to left). WC denote by $\mathcal{L}_{Y}(X)$ the ring of all continuouss linear operators of $X$ and by $\mathcal{F}_{Y}(X)$ the ideal of those operators having finite rank.

The subrings of $\mathcal{L}_{Y}(X)$ containing $\mathcal{F}_{Y}(X)$ are precisely those primitive (equivalently, prime) rings with nonzero socle. One can see that such rings are algebras over $K$ when $\Delta$ is a $K$-algebra. By [7, Theorcm 1], the symmetric Martindale algebra of quotients of a prime associative algebra $A$ with nonzero socle:

$$
\mathcal{F}_{Y}(X)^{+}=\operatorname{Soc}(A) \mathrm{C} A \subset \mathcal{L}_{Y}(X)
$$

is precisely $Q(A)=\mathcal{L}_{Y}(X)$.

If $A$ has an involution then $\Delta$ has an involution, $X$ is self-dual with respect to a hermitian or alternating inner product $\langle.,$.$\rangle , and the involu-$ tion is the adjoint with respect to this inner product. In the alternating case $\Delta$ is a field $F$ and the identity is its involution.

(4.2) Theorem (Osborn-Racine). Let $J$ be a prime nondegenerate Jordan algebra with nonzero socle. Then one of the following conditions holds:

(a) $J$ is a simple 27-dimensional exceptional Jordan algebra over its centre,

(b) $J$ is a simple Jordan algebra of a nondegenerate symmetric bilinear form,

(c) there exists a pair of dual vector spaces $(X, Y,(.)$.$) over a division$ associative algebra $\Delta$ such that

$$
\mathcal{F}_{Y}(X)^{+}=\operatorname{Soc}(J) \subset J \subset \mathcal{L}_{Y}(X)^{+}=Q\left(\mathcal{F}_{Y}(X)\right)^{+},
$$


(d) there exists a hermitian or alternating self-dual vector space $(X,\langle, .$,$) such that$

$$
\begin{gathered}
H\left(\mathcal{F}_{X}(X), *\right)=\operatorname{Soc}(J) \subset J \subset H\left(\mathcal{L}_{X}(X), *\right)=H\left(Q\left(\mathcal{F}_{X}(X)\right), *\right) \\
\text { where } *: \mathcal{L}_{X}(X) \rightarrow \mathcal{L}_{X}(X) \text { denotes the adjoint involution. }
\end{gathered}
$$

(4.3). Since any simple Jordan algebra is prime and nondegenerate, we get in particular that every simple Jordan algebra $J$ containing minimal inner ideals is either exceptional, quadratic, $\mathcal{F}_{Y}(X)^{+}$or $H\left(\mathcal{F}_{X}(X), *\right)$.

Let $(X, Y,(,)$,$) be a pair of dual vcctor spaces over \Delta$. For each $x \in X, y \in Y$ write $y \otimes x$ to denote the continuous linear mapping of $X$ defined by

$$
x^{\prime}(y \otimes x)=\left\langle x^{\prime}, y\right\rangle x, \text { for all } x^{\prime} \in X .
$$

(4.4). $\mathcal{F}_{Y}(X)$ is generated as an abelian group by all these operators.

The following fundamental result on dual pairs will be frccuently used in what follows $[19, \mathrm{p} .119]$.

(4.5). Let $(X, Y,(,)$,$) be a pair of dual vector spaces over \Delta$. Let $x_{1}, \ldots, x_{n} \in X$ be lineorly independent. Then there exist $y_{1}, \ldots, y_{n} \in Y$ such that $\left(x_{i}, y_{i}\right)=1$ for all $i,\left(x_{i}, y_{j}\right)=0$ for $i \neq j$.

We remark that every hermitian or alternating self-dual vector space $(X,\langle,\rangle$,$) over (\Delta,-)$ gives rise to a pair of dual vector spaces $(X, X,(, .)$,$) where the second X$ is regarded as a right vector space over $\Delta$ by defining $x . \alpha=\ddot{\alpha} x$. It is not difficult to verify the following two statements.

(4.6). If $(X,\langle, .\rangle$,$) is hermitian then (y \otimes x)^{*}=x \otimes y$, and $J=$ $H\left(\mathcal{F}_{X}(X), *\right)$ is generated as an abelian group by the operators of the form $x \otimes \alpha x, y \otimes z+z \otimes y$, for all $x, y, z \in X, \alpha \in H(\Delta,-)$.

(4.7). If $(X,\langle, .)$,$) is aliernating then (y \otimes x)^{*}=-x \otimes y$ and $J=$ $H\left(\mathcal{F}_{X}(X), *\right)$ is generated as an abelian group by the operators of the form $y \otimes x-x \otimes y$.

(4.8) Proposition. Let $(X,\langle, .\rangle$,$) be a self-dual vector space. Then$ $\left(\mathcal{F}_{X}(X), *\right)$ is a $*$-envelope for $J=H\left(\mathcal{F}_{X}(X), *\right)$ if $(X,\langle,\rangle$.$) is her-$ mitian over a division associative algebra with involution $(\Delta,-)$ with 
$\operatorname{dim}_{\Delta} X>1$, or if $(X,\langle, .\rangle$,$) is alternating over a field F$ with $\operatorname{dim}_{F} X>$ 2 .

Proof: Let $A$ denote the associative algebra (over $K$ ) generated by $J=H\left(\mathcal{F}_{X}(X), *\right)$.

Suppose first that $(X,\langle,\rangle$,$) is hermitian.$

(i) If $\langle x, y\rangle=1$ then $(x, \otimes x)(y \otimes y\rangle=x \otimes\langle x, y\rangle=x \otimes y \in A$ by (4.6).

(ii) Suppose now that $x, y$ are linearly independent. By (4.5), there exists $z \in X$ such that $\langle x, z\rangle=0,\langle y, z\rangle=1$. Hence, by (i), $z \otimes y \in A$, so by (4.6) again $(y \otimes x+x \otimes y)(z \otimes y)=x \otimes y \in A$.

(iii) Finally we must prove that $x \otimes \alpha x \in A$ for all $\alpha \in \Delta$. We may assume that $x=x_{1}$ is nonzero. Since $\operatorname{dim}_{\Delta} X>1$ there is $x_{2} \in X$ such that $x_{1}, x_{2}$ are linearly independent. Take, by (4.5), $y_{1}, y_{2} \in X$ satisfying $\left\langle x_{i}, y_{j}\right\rangle=\delta_{i j}$. By (ii) and (4.6), $\left(x_{1} \otimes \alpha x_{2}\right)\left(y_{2} \otimes y_{1}\right)\left(x_{1} \otimes x_{1}\right)=x \otimes \alpha x \in A$.

Suppose now that $(X,\langle, .\rangle$,$) is alternating.$

(iv) If $\langle x, y\rangle=0$ with $x, y$ linearly independent take, by (4.5), $x^{\prime} \in X$ such that $\left\langle x^{\prime}, x\right\rangle=1,\left\langle y, x^{\prime}\right\rangle=0$. Then, by (4.7),

$$
\left(x \otimes x^{\prime}+x^{\prime} \otimes x\right)(x \otimes y-y \otimes x)=x \otimes y \in A .
$$

(v) Since $\operatorname{dim}_{F}>2$, given a nonzero vector $x \in X$, there exists $y \in Y$ such that $\langle x, y\rangle=0$ with $x, y$ linearly independent. Hence, by (iv), for each $\alpha \in \Delta$ we have $y \otimes \alpha x \in A$. Take $y^{\prime} \in X$ such that $\left\langle y^{\prime}, y\right\rangle=1$. Then

$$
\left(x \otimes y^{\prime}+y^{\prime} \otimes x\right)(y \otimes \alpha x)=x \otimes \alpha x \in A .
$$

(vi) Suppose finally that $x, y$ are linearly independent and take $x^{\prime} \in X$ such that $\left\langle x, x^{\prime}\right\rangle=1,\left\langle y, x^{\prime}\right\rangle=0$. By (iv), (v), $x^{\prime} \otimes y, x \otimes x \in A$. Then $(x \otimes y)\left(x^{\prime} \otimes y\right)=x \otimes y \in A$, which completes the proof.

Theorem. Let $J$ be a prime nondegenerate Jordan algebra with nonzero socle of hermitian type, $S \subset J$, and $a_{1}, a_{2} \in \operatorname{Soc}(J)$.

If $(1) \mathcal{F}_{Y}(X)^{+}=\operatorname{Soc}(J) \subset J \subset \mathcal{L}_{Y}(X)^{+}=Q\left(\mathcal{F}_{Y}(X)\right)^{+}$where $(X, Y,(\cdot)$,$) is a pair of dual vector spaces over \Delta$. Then

$$
\operatorname{Ran}_{\mathcal{L}_{Y}(X)}(S)=\left\{b \in \mathcal{L}_{Y}(X): X S \subset \operatorname{ker}(b)\right\}
$$

$$
\operatorname{Lan}_{\mathcal{L}_{Y}(X)}(S)=\left\{c \in \mathcal{L}_{Y}(X): S^{\#} Y \subset \operatorname{ker}\left(c^{\#}\right)\right\}
$$

$$
\operatorname{Ann}_{J}(S)=\left\{b \in J: X S \subset \operatorname{ker}(b), S^{\#} Y \subset \operatorname{ker}\left(b^{\#}\right)\right\},
$$

$$
\operatorname{Ann}_{J}\left(a_{1}\right) \subset \operatorname{Ann}_{J}\left(a_{2}\right) \text { if and only if } X a_{2} \subset X a_{1} \text { and } a_{2}^{\#} Y \subset a_{1}^{\#} Y \text {. }
$$


If (2) $H\left(\mathcal{F}_{X}(X), *\right)=\operatorname{Soc}(J) \mathrm{C} J \subset H\left(\mathcal{L}_{X}(X), *\right)=H\left(Q\left(\mathcal{F}_{X}(X)\right), *\right)$ where $(X,\langle.,$.$) is a hermitian or alternating self-dual vector space, then$

$$
\operatorname{Ann}_{J}(S)=\operatorname{Ran}_{\mathcal{L}_{Y}(X)}(S) \cap J=\{b \in J: X S \subset \operatorname{ker}(b)\}, \text { and }
$$

$\operatorname{Ann}_{J}\left(a_{1}\right) \subset \operatorname{Ann}_{J}\left(a_{2}\right)$ if and only if $X a_{2} \subset X a_{1}$.

Proof: (4.9) follows from definition, while (4.10) is a consequence of (4.9) since $\operatorname{Lan}(S)=\operatorname{Ran}\left(S^{\#}\right)^{\#}$, where $b \rightarrow b^{\#}$ is the canonical anti-isomorphism of $\mathcal{L}_{Y}(X)$ onto $\mathcal{L}_{X}(Y)$ (associated to the dual pair $\left(Y, X,(\ldots)^{\mathrm{op}}\right)$

(4.11) Since $Q\left(\mathcal{F}_{Y}(X)\right)=\mathcal{L}_{Y}(X)$, we have by (3.6) that $\operatorname{Anm}_{J}(S)=$ $\operatorname{Ann}_{\mathcal{L}_{Y}(X)}(S) \cap J$, and hence (4.11) follows from (4.9) and (4.10).

(4.12) By (4.11), $X a_{2} \subset X a_{1}$ and $a_{2}^{\#} Y \subset a_{1}^{\#} Y$ imply $\operatorname{Ann}_{J}\left(a_{1}\right) \subset$

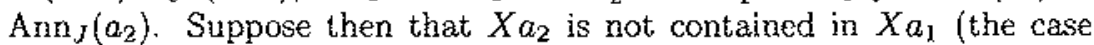
that $a_{2}^{\#} Y$ is not contained in $a_{1}^{\#} Y$ would follow by symmetry). Since $X a_{1}$ is a proper subspace of $X, a_{1}^{\#} Y$ is also proper because $a_{1}$ and $a_{1}^{\#}$ have the same (finitc) rank. Hence, by (4.5), there exists $y \in Y$ such that $\left\langle X a_{1}, y\right\rangle=0$ but $\left\langle X a_{2}, y\right\rangle \neq 0$. Similarly we can take $0 \neq x \in X$ such that $\left\langle x, a_{1}^{\#} Y\right\rangle=0$. Set $c=y \otimes x \in \mathcal{F}_{Y}(X)$. Then

$$
\begin{aligned}
X a_{1}(y \otimes x)=\left\langle X a_{1}, y\right\rangle x=0 & \Rightarrow X a_{1} \subset \operatorname{ker}(c) \\
& \Rightarrow(\text { by } 4.9) c \in \operatorname{Ran}_{\mathcal{C}_{Y}(X)}\left(a_{1}\right)
\end{aligned}
$$

and

$$
\begin{aligned}
c^{\#}\left(a_{1}^{\#} Y\right)=y\left\langle x, a_{1}^{\#} Y\right\rangle=0 & \Rightarrow a_{1}^{\#} Y \subset \operatorname{ker}\left(c^{\#}\right) \\
& \Rightarrow(\text { by } 4.10) c \in \operatorname{Ran}_{\mathcal{L}_{Y}(X)}\left(a_{1}\right) .
\end{aligned}
$$

Therefore $c \in \operatorname{Ann}_{J}\left(a_{1}\right)$; but $c$ does not lie in $\operatorname{Ann}_{J}\left(a_{2}\right)$ because $\left\langle X a_{2}, y\right\rangle \neq 0$.

Suppose now that $J$ is as in (2). If $(X,\langle.,$.$) is hermitian over a di-$ vision associative algebra with involution $(\Delta,-)$ with $\operatorname{dim}_{\Delta} X=1$, or alternating over a field $F$ with $\operatorname{dim}_{F} X=2$, then $J=H\left(\mathcal{L}_{X}(X), *\right)$ is a division Jordan algebra, and hence (4.13) holds trivially in this case. Suppose then that $(X,\langle.,\rangle$.$) is hermitian over a division associative alge-$ bra, with involution $(\Delta,-)$ with $\operatorname{dim}_{\Delta} X>1$, or alternating over $F$ with $\operatorname{dim}_{F} X>2$. By $(4.8) H\left(\mathcal{F}_{X}(X), *\right)$ generates $\mathcal{F}_{X}(X)$ as an associative algebra, and hence, by (3.6),

$$
\begin{aligned}
\operatorname{Ann}_{J}(S) & =J \cap \operatorname{Ann}_{\mathcal{L}_{Y}(X)}(S)= \\
& =J \cap \operatorname{Ran}_{\mathcal{C}_{Y}(X)}(S)=\{b \in J: X S \subset \operatorname{ker}(b)\}
\end{aligned}
$$


since $\mathcal{L}_{X}(X)=Q\left(\mathcal{F}_{X}(X)\right)$, which proves (4.13).

(4.14) By (4.13), $X a_{2} \subset X a_{1}$ implies that $\operatorname{Ann}_{J}\left(a_{1}\right) \subset \operatorname{Ann}_{J}\left(a_{2}\right)$. Conversely, suppose that $X a_{2}$ is not contained in $X a_{1}$. If the inner product $\langle.,$.$\rangle is hermitian, take, by (4.5), y \in X$ such that $\left\langle X a_{1}, y\right\rangle=0$ but $\left\langle X a_{2}, y\right\rangle \neq 0$. Then $b=y \otimes y$ belongs to $\operatorname{Ann}_{J}\left(a_{1}\right)$ but does not belong to $\mathrm{Ann}_{J}\left(a_{2}\right)$ by (4.13). If the inner product is alternating the proof is slightly more complicated. We first note that if $X$ is finitedimensional then the rank of every operator $a \in H\left(\mathcal{F}_{X}(X), *\right)$ is even. Actually this is true even if $X$ is infinite-dimensional. Hence, if $X a_{2}$ is not contained in $X a_{1}$ then $X a_{1}$ is a proper subspace of $X$ with $\operatorname{codim}\left(X a_{1}\right) \geq$ 2. Then, by (4.5), there exist $y, z$ linearly independent in $X$ such that

$$
\left\langle X a_{1}, y\right\rangle=0=\left\langle X a_{1}, z\right\rangle
$$

but

$$
\left\langle X a_{2}, y\right\rangle \neq 0 \text {. }
$$

Take $b=y \otimes z-z \otimes y \in H\left(\mathcal{F}_{X}(X), *\right)$. Then $X a_{1} \subset \operatorname{ker}(b)$ and hence $b \in \operatorname{Ann}_{J}\left(a_{1}\right)$ by (4.13). However, $b$ does not belong to $\operatorname{Ann}_{J}\left(a_{2}\right)$ since $\left\langle x a_{2}, y\right\rangle \neq 0$ for a certain $x \in X$ implies

$$
\left(x a_{2}\right) b=\left\langle x a_{2}, y\right\rangle z-\left\langle x a_{2}, z\right\rangle y \neq 0
$$

because $z, y$ are linearly independent.

(4.15) Theorem. A nondegenerate Jordan algebra $J$ satisfying the descending chain condition on the principal inner ideals, also satisfies the ascending chain condition on the annihilators of the principal inner ideals.

Proof: By [8] (sce also [14]), $J$ coincides with its socle, and hence it is a direct sum of simple ideals. This reduces the question to the case that $J$ is simple. Then by applying Osborn-Racine theorem we can consider the following cases:

Case $1 . J$ is a simple exceptional finite dimensional Jordan algebra over its centre $Z(J)$.

Since annihilators are irvariant under multiplications by elements of the centre, they are subspaces of a finite-dimensional vector space, so they satisfy the maximality condition.

Case 2. $J=F 1 \oplus V$ is the simple Jordan algebra of a nondegenerate symmetric bilinear form $\langle.,$.$\rangle on a vector space V$ with $\operatorname{dim}_{F} V \geq 2$.

Let $a=\alpha+x \in J$. If $\alpha^{2}-\langle x, x\rangle \neq 0$ then we have by (1.22) that $a$ is invertible, so $\operatorname{Ann}\left(U_{0} J\right)=\operatorname{Ann}(J)=0$. Suppose then that $\alpha^{2}-\langle x, x\rangle=$ 
0 . If $\alpha \neq 0$ then by (2.10) $\operatorname{Ann}\left(U_{a} J\right)=\operatorname{Ann}(a)=F(\alpha-x)$, while if $\alpha=0$ then $\operatorname{Ann}\left(U_{a} J\right)=\operatorname{Ann}(a)=F x$ whenever $a=x \neq 0$. Therefore $J$ satisfies the maximality condition on the annihilators of the principal inner ideals.

Case 3. $J=\mathcal{F}_{Y}(X)^{+}$where $(X, Y,\langle.,)$.$) is a pair of dual vector$ spaces over a division associative algebra $\Delta$.

By (4.12), Ann $\left(a_{1}\right) \subset \operatorname{Ann}\left(a_{2}\right)$ implies that

$$
X a_{1} \supset X a_{2} \supset \ldots
$$

and

$$
a_{1}^{\#} Y \supset a_{2}^{\#} Y \supset \ldots
$$

Since $X a_{1}$ and $a_{1}^{\#} Y$ are finite-dimensional, both descending chains are stationary. Hence, by (4.12) again, $\operatorname{Ann}\left(U_{a_{n}} J\right)=\operatorname{Ann}\left(a_{n}\right)=$ $\operatorname{Ann}\left(a_{m}\right)=\operatorname{Ann}\left(U_{a_{m}} J\right)$ for some positive integer $n$ and all $m \geq n$.

Case 4. $J=H\left(\mathcal{F}_{X}(X), *\right)$ where $(X,\langle, .)$,$) is a hermitian or al-$ ternating self-dual vector space over a division associative algebra with involution $(\Delta,-)$.

It is similar to Case 3 since, by (4.14), $\operatorname{Ann}\left(U_{a_{1}} J\right)=\operatorname{Ann}\left(a_{1}\right) \subset$ $\operatorname{Ann}\left(a_{2}\right)=\operatorname{Ann}\left(U_{a_{2}} J\right)$ if and only if $X a_{2} \subset X a_{1}$.

(4.16) Remark. We note that a nondegenerate Jordan algebra $J$ coinciding with its socle need not satisfy the descending chain condition on the annihilators of the principal inner ideals. Indeed, let $J$ be a simple Jordan algebra without identity element and which contains minimal inner ideals. Then, by [17, Cor. 7$], J$ contains an infinite sequence $\left\{e_{n}\right\}$ of nonzero orthogonal idempotents. Hence

$$
\operatorname{Ann}\left(U_{e_{1}} J\right) \supset \operatorname{Ann}\left(U_{e_{1}+e_{2}} J\right) \supset \operatorname{Ann}\left(U_{e_{1}+e_{2}+e_{3}} J\right) \supset \ldots
$$

where the inclusions are strict. Since $\operatorname{Ann}(\operatorname{Ann}(\operatorname{Ann}(M)))=\operatorname{Ann}(M)$ for every subset $M$ of $J$, we also have that such an algebra $J$ does not satisfy neither the descending chain condition nor the ascending chain condition on the annihilators of arbitrary subsets.

\section{Annihilators in anisotropic Jordan algebras and in $J B$-algebras}

A real Jordan $J$ with a complete norm, $\|$.$\| such that, for all elements$ $a, b$ in $J$
(i) $\|a . b\| \leq\|a\|\|b\|$,
(ii) $\|a\|^{2} \leq\left\|a^{2}+b^{2}\right\|$ 
is called a $J B$-algebra. The set $J^{2}$ of all squares of elements in a $J B$ algebra $J$ is a proper convex cone. Therefore $J^{2}$ induces an ordering on $J$. For every element $a \in J$ the mapping $U_{a}: J \rightarrow J$ is positive, i.e., $U_{a} J^{2} \subset J^{2}$. The reader is referred to [10] for general results on $J B$-algebras. From (i), (ii) we get

$$
\text { (iii) }\|a\|^{2}=\left\|a^{2}\right\| \text {. }
$$

Hence $J B$-algebras are anisotropic, i.e., they do not contain nonzero nilpotent elements.

Let $J$ be an arbitrary Jordan algebra. For any subset $T$ of $J$, write

$$
\begin{aligned}
q(T) & =\left\{a \in J: U_{T} a^{2}=0\right\} \\
q^{\prime}(T) & =\left\{a \in J: U_{a} T^{2}=0\right\} \\
T^{0} & =\{a \in J: a \cdot T=0\} \\
T^{\perp} & =\left\{a \in J: a^{2} \cdot T=0\right\}
\end{aligned}
$$

Always we have the following inclusion

$$
\operatorname{Ann}(T) \subset q(T) \cap q^{\prime}(T) \cap T^{0} \cap T^{\perp}
$$

Indeed, since $A n n(T)$ is an strict inner ideal by (2.4), $a \in \operatorname{Ann}(T) \Rightarrow$ $a^{2} \in \operatorname{Ann}(T)$. Hence $a \cdot T=a^{2} \cdot T^{2}=U\left(a^{2}\right) T=U_{T} a^{2}=0$.

Theorem. Let $J$ be an anisotropic Jordan algebra. Then

$$
q(T)=q^{\prime}(T)=\operatorname{Ann}(T)=T^{0} \cap T^{\perp} \text { for every subset } T \text { of } J
$$

$$
\operatorname{Ann}(x)=\operatorname{Ann}\left(x^{2}\right) \text { for all } x \in J
$$

$$
\operatorname{Ann}(I)=I^{0}=I^{\perp}=q(I)=q^{\prime}(I) \text { for every inner ideal I of } J .
$$

Proof: Let $T$ be a subset of $J$. Then

$$
q(T)=q^{\prime}(T)
$$

since in the abscense of nilpotent elements $U_{x} y^{2}=0 \Rightarrow\left(U_{y} x^{2}\right)^{2}=$ $U_{y} U_{x} U_{x} y^{2}=0$

$$
a \in q(T)=q^{\prime}(T) \Rightarrow U_{a} T=U_{T} a=0
$$


because $\left(U_{y} x\right)^{2}=U_{y} U_{x} y^{2}$

$$
a \in q(T)=q^{\prime}(T) \Rightarrow a \cdot T=a^{2} \cdot T=0
$$

since by (1.13), $4(a . t)^{2}=U_{a} t^{2}+U_{t} a^{2}+2 a . U_{t} a=0$ by $(2)$, and $q^{\prime}(T)$ is an strict inner subset: $\left(a \in q^{\prime}(T) \Rightarrow U_{a} J^{1} \subset q^{\prime}(T) \Rightarrow a^{2} \in q^{\prime}(T)\right)$.

Since, by $(5.1), \operatorname{Ann}(T) \subset q(T)$, it follows from (3)

$$
\operatorname{Ann}(T) \subset q(T)=q^{\prime}(T) \subset T^{0} \cap T^{\perp}
$$

Suppose now that $I$ is an inner ideal of $J$. Then $t \in I \Rightarrow U_{t} J \subset I$, and hence $a \in I^{0} \Rightarrow a . I=a . U_{I} a=0$, so by (1.17)

$$
U_{a} t^{2}=2\{a . t t a\}-a . U_{t} a=0 .
$$

Since $q^{\prime}(I) \subset I^{0}$ by (4), we have proved

$$
r^{0}=q(I)=q^{\prime}(I)
$$

By linearizing (1.12) we get

$$
\begin{aligned}
4\{a t x\}^{2}= & U_{a} U_{t} x^{2}+U_{x} U_{t} a^{2}-2 U_{a} t . U_{x} t+4\left\{a U_{t}(a, x) x\right\}=(\text { by } 1.18) \\
& U_{a} U_{t} x^{2}+U_{x} U_{t} a^{2}-2 U_{a} t . U_{x} t+8\{a\{a . t x t\} x\}-4\left\{a a . U_{t} x x\right\}
\end{aligned}
$$

Hence, $a \in I^{0} \Rightarrow\left\{a I J^{1}\right\}^{2}=0$ (because $U_{I} a^{2}=U_{a} I=0$ by (5), (2)) $\Rightarrow\left\{a I J^{1}\right\}=0$ implies $a \in \operatorname{Ann}(I)$. Since Ann $(I) \subset I^{0}=q(I)=q^{\prime}(I)$ by $(5.1),(5)$, we have proved

$$
\operatorname{Ann}(I)=I^{0}=q(I)=q^{\prime}(I)
$$

Now let $x \in J$. Always $\operatorname{Ann}(x) \subset \operatorname{Ann}\left(x^{2}\right)$, and in the abscense of nilpotent elements, $y \in \operatorname{Ann}\left(x^{2}\right) \Rightarrow U_{y} x^{2}=0 \Rightarrow x \in q\left(\operatorname{Ann}\left(x^{2}\right)\right)=$ (by 6) Ann(Ann $\left.\left(x^{2}\right)\right)$ since $A n n\left(x^{2}\right)$ is an inner ideal. Then, by (2.5), $x \in \operatorname{Ann}\left(\operatorname{Ann}\left(x^{2}\right)\right) \Rightarrow \operatorname{Ann}\left(x^{2}\right) \subset \operatorname{Ann}(x)$, which proves 


$$
\operatorname{Ann}(x)=\operatorname{Ann}\left(x^{2}\right)
$$

Now $a \in I^{\perp} \Rightarrow a^{2} . I=0 \Rightarrow$ (by 6$) a^{2} \in I^{0}=\operatorname{Ann}(I) \Rightarrow I \subset \operatorname{Ann}\left(a^{2}\right)=$ Ann(a) by (7). Hence $I^{\perp} \subset \operatorname{Ann}(I)$. Since $\operatorname{Ann}(I)$ is contained in $I^{\perp}$ by $(5.1)$, we get from $(6)$

$$
\operatorname{Ann}(I)=I^{0}=I^{\perp}=q(I)=q^{\prime}(I) .
$$

Turn to the general case of a subset $T$ of $J$. Then $a \in T^{0} \cap T^{\perp} \Rightarrow a \cdot T=$ $a^{2} \cdot T=0 \Rightarrow$ (by 2.9) $a^{2} \in \operatorname{Ann}(T) \Rightarrow T \subset \operatorname{Ann}\left(a^{2}\right)=\operatorname{Ann}(a) \Rightarrow a \in$ Ann(T). Since Ann $(T) \subset q(T)=q^{\prime}(T) \subset T^{0} \cap T^{\perp}$ by (4), we get

$$
q(T)=q^{\prime}(T)=\operatorname{Ann}(T)=T^{0} \cap T^{\perp}
$$

which completes the proof of the theorem.

(5.5) Corollary. If $e$ is an idempotent in an anisotropic Jordan algebra $J$ then

$$
\operatorname{Ann}(e)=q(\{e\})=q^{\prime}(\{e\})=\{e\}^{0}=\{e\}^{\perp} .
$$

Proof: By (5.2), Amn $(e)=q(\{e\})=q^{\prime}(\{e\})=\{e\}^{0} \cap\{e\}^{\perp}$. Since $\operatorname{Ann}(e)=\{e\}^{0}$ by (2.11), $a \in\{e\}^{\perp} \Rightarrow a^{2} . e=0 \Rightarrow e \in \operatorname{Ann}\left(a^{2}\right)=\operatorname{Ann}(a)$ by (5.3), $\Rightarrow a \in \operatorname{Ann}(e)$, which completes the proof.

In a recent paper [2], Battaglia proved the inclusion $T^{\perp} \subset T^{0}$ for any subset $T$ of a $J B$-algebra. The proof of this result uses the fundamental fact (proved by the author in the same paper) that if $a^{2} . b=0$ then $a^{2} \cdot b^{2}$ is a positive clement. Indeed, $a \in T^{\perp} \Rightarrow a^{2} \cdot T=0$, and hence $U_{t} a^{2}=-t^{2} \cdot a^{2}\left(t \in T^{\prime}\right)$. Since $U_{t}: J \rightarrow J$ is a positive mapping, $U_{t} a^{2}=0$, and hence $a \in q(T) \subset T^{0}$. From this result and from (5.2) we get have

(5.6) Corollary. Let $J$ be a. $J B$-algebra. For every subset $T$ of $J$ we

$$
q(T)=q^{\prime}(T)=\operatorname{Ann}(T)=T^{\perp} .
$$

Clearly, if an element $x$ in a Jordan algebra. $J$ has annihilator different from zero, then this element is a zero divisor, i.e., $U_{x}: J \rightarrow J$ is not injective. The converse is true in $I B$-algebras.

(5.7) Proposition. Let $J$ be a $J B$-algebra. Then every zero divisor $x$ in $J$ has nonzero annihilator. 
Proof: Let $U_{x} y=0$ for some nonzero $y \in J$. Then $0=\left(U_{x} y\right)^{2}=$ $U_{x} U_{y} x^{2}$ and we have two possibilities:

(1) If $U_{y} x^{2}=0$ then $y \in q^{\prime}(\{x\})=\operatorname{Ann}(x)$ by (5.2).

(2) If $U_{y} x^{2} \neq 0$ then $U_{y} x^{2}=z^{2}$ for some nonzcro $z \in J$ because the mapping $U_{y}: J \rightarrow J$ is positive. Hence, $0=U_{x} z^{2} \Rightarrow z \in q(\{x\})=$ $\operatorname{Ann}(x)$ by (5.2). In both cascs $\operatorname{Ann}(x) \neq 0$ as required.

(5.8) Remark. (i) As it is shown in [2], the inclusion $T^{0} \subset T^{i}$ can be strict even in a $J B$-algebra.

(ii) The equality $q(I)=I^{0}$ was first obtained in [5, Lemma 1i] for normed closed ideals in $J B$-algebras, and later this result was extended in [6. Lemma 6.8] to inner ideals in annisotropic Jordan algebras.

(iii) The equaliiy $q(\{e\})=\{e\}^{n}=\{e\}^{\perp}$ was proved in [13, Lemma 11] for an idempotent in a unital $J B$-algebra.

I wish to thank Prof. Rodríguez Palacios and my colleagues Eulalia García Rus, Esperanza Sánchez Campos and Mercedes Siles Molina for their suggestions which have improved this paper.

\section{References}

1. P. N. ANH, Simple Jordan algcbras with minimal inner ideals, Commun. in Algebra 14 (3) (1986): 489-492.

2. M. BatTaglia, Annihilators in $J B$-algebras, Math. Proc. Camb. Phil. Soc. 108 (1990), 317-323.

3. K. I. Beidar, $A$. V. Mikhalev and A. M. Silinko, Criteria for primeness of nondegenerate alternative and Jordan algebras, Tran. Moscow Math. Soc. (1988), 129-137.

4. M. Bresar, Jordan derivations on semiprime rings, Proc. Amer. Math. Soc. 104 (2) (1988), 1003-1006.

5. L. J. Bunce, The theory and structure of dual $J / 3$-algebras, Math. Z. $180(1982), 525 \cdot 534$.

6. A. FERNÁNDEZ LÓPEZ, Modular annihilators Jordan algebras, Commun, in Algebra 13 (12) (1985), 2597-2613.

7. A. FER.NÁYide: LÓPEz, Prime nondegenerate Jordar algebras with nonzero socle and the symmetric Martindale algebra or quotients, Collectanea Moith. 39 (1988), 249-256.

8. A. FERnÁNDEZ LÓpeZ AND E. GARCía RL'S, A characterization of the elements of the socle of a Jordan algcbras, Proc. Amer. Math. Soc. 108 (1990), 69-71. 
9. A. Fernández López and A. Rodríguez Palacios, On the socle of a non-commutative Jordan algebra, Monuscripta Moth. 56 (1986), 269-278.

10. H. HANChE-OLSEN AND E. STøRMER, "Jordan operators algebras," Monographs. Stud. Math. 21, Pitman, 1984.

11. N. Jacobson, "Structure of rings," Colloq. Publ. 37, Amer. Math. Soc. Providence R.I., 1964.

12. N. JACOBSON, "Structure and representations of Jordan algebras," Colloq. Publ. 39, Amer. Math. Soc. Providence R.I., 1968.

13. P. Jiménez Garijo and A. Rodríguez Palacios, The Jordan regular ring associated to a finite $J B W$-algebra, $J$. Algebra 110 (1) (1987), 56-76.

14. O. Loos, On the socle of a Jordan pair, Collectanea Math. 40 (2) (1989), 109-125.

15. K. MCCrimmon, Martindale systems of symmetric quotients, Algebra, Groups and Geometries 6 (1989), 153-237.

16. K. MCCRIMmon AND E. ZeL'MANov, The structure of strongly prime quadratic Jordan algebras, Adv. in Math. 69 (1988), 133-222.

17. J. M. OsBorn and M. L. RACine, Jordan rings with nonzero socle, Trans. Amer. Math. Soc. 251 (1979), 375-387.

18. D. S. PASSMAN, Computing the symmetric rings of quotients, $J$. Algebra 105 (1987), 207-235.

19. P. Ribenboim, "Rings and modules," Interscience Tracts. in Pure and Applied Math. 24, John Wiley \& Sons, 1969.

20. D. M. TopPING, "Jordan algebras of self-adjoints operators," Memoirs Amer. Math. Soc. 53, Amer. Math. Soc., 1965.

21. E. Zel'manov, Jordan algebras with finiteness conditions, Algebra i Logika 17 (1978), 693-704.

22. E. Zel'manov, Jordan division algebras, Algebra i Logika 18 (1979), 286-310.

23. E. Zel'MANov, On prime Jordan algebras II, Siberian Math. J. 24 (1983), 89-104.

24. E. ZEL'MANOV, Goldie's theorem for Jordan algebras, Siberian Math. J. 28 (1987), 44-52. 
25. K. A. Zhevlakov, A. M. Slinko, I. P. Shestakov and A. I. SHIRSHOV, "Rings that are nearly associative," Academic Press, 1982.

Departamento de Álgebra, Geometria y Topología

Facultad de Ciencias

Universidad de Málaga

Campus Teatinos

29071 Málaga

SPAIN

Primera versió rebuda el 7 de Gener de 1992, darrera versió rebuda el 15 de Febrer de 1992 